Association for Information Systems AIS Electronic Library (AISeL)

Wirtschaftsinformatik Proceedings 2003

Wirtschaftsinformatik

September 2003

\title{
Ein Kommunikationssystem zur Unterstützung der mobilen Kommunikation in der Speditionslogistik
}

Volker Gruhn

Universität Leipzig

Lothar Schöpe

Informatik Centrum Dortmund e.V., lothar.schoepe@uni-dortmund.de

Follow this and additional works at: http://aisel.aisnet.org/wi2003

\section{Recommended Citation}

Gruhn, Volker and Schöpe, Lothar, "Ein Kommunikationssystem zur Unterstützung der mobilen Kommunikation in der Speditionslogistik" (2003). Wirtschaftsinformatik Proceedings 2003. 8.

http://aisel.aisnet.org/wi2003/8

This material is brought to you by the Wirtschaftsinformatik at AIS Electronic Library (AISeL). It has been accepted for inclusion in Wirtschaftsinformatik Proceedings 2003 by an authorized administrator of AIS Electronic Library (AISeL). For more information, please contact elibrary@aisnet.org. 
In: Uhr, Wolfgang, Esswein, Werner \& Schoop, Eric (Hg.) 2003. Wirtschaftsinformatik 2003: Medien - Märkte - Mobilität, 2 Bde. Heidelberg: Physica-Verlag

ISBN: 3-7908-0111-9 (Band 1)

ISBN: 3-7908-0116-X (Band 2)

(C) Physica-Verlag Heidelberg 2003 


\title{
Ein Kommunikationssystem zur Unterstützung der mobilen Kommunikation in der Speditionslogistik
}

\author{
Volker Gruhn \\ Universität Leipzig \\ Lothar Schöpe \\ Informatik Centrum Dortmund e.V.
}

Zusammenfassung: Der Einsatz von mobiler Kommunikation bei Speditionsunternehmen zur Unterstützung von Logistikprozessen ist nicht erst zur Einführung der elektronischen Maut Gegenstand von F\&E Aktivitäten bei Unternehmen und Forschungseinrichtungen. Von Softwareunternehmen werden die unterschiedlichsten Kommunikationssysteme angeboten, die sich hinsichtlich verwendeter Kommunikationstechnik (Bündelfunk, GSM), ihrer Kommunikationsarchitektur (zentral, dezentral), ihrer Anwendung (SMS, WAP), der Art der genutzten Informationen (Sensorikdaten, Telematikdaten, Transportdaten, Dispositionsdaten) und dem Grad der Integration mit Speditionslogistikanwendungen unterscheiden. Durch den Einsatz von neuen Kommunikationstechniken (GPRS, HSCSD, UMTS) und Telematiktechniken (GPS, LBS) sowie Softwaretechniken (C\#, .NET, WebServices) könnten verteilte Client/Serveranwendungen mit umfangreicher verteilter Geschäftslogik zur Unterstützung der Speditionslogistik zum Einsatz kommen.

In dem Verbundforschungsprojekt „Mobile Spedition im Web (SpiW) “" werden diese neuen Techniken zur Entwicklung eines Kommunikationssystems zur Unterstützung der Speditionslogistik eingesetzt.

In diesem Beitrag wird das Verbundforschungsprojekt sowie dessen initiale Motivation und Problemstellung beschrieben. Die Art der Entwicklung des Kommunikationssystems und dessen Architektur wird ebenso dargestellt wie eine exemplarische Anwendung.

Schlüsselworte: mobile Kommunikation, Speditionslogistik, Client/Server

\footnotetext{
Gefördert durch das BMBF unter dem Kennzeichen 01HT0143.
} 


\section{Einleitung}

Speditionsunternehmen erzielen einen Wettbewerbsvorsprung, wenn sie durch einen funktionierenden Güterverkehr Transporte schnell und sicher sowie pünktlich und kostengünstig durchführen können. „Die Zeit wird im Straßengüterverkehr mehr und mehr zu einer kritischen Komponente " [ErWa97]. Dieser Wettbewerbsvorsprung ist um so wichtiger, da durch den Wegfall der Tarifbindung, des Kabotageverbots und der Globalisierung die Anzahl der Mitbewerber am Markt steigt. Speditionsunternehmen, die durch den Einsatz von mobiler Kommunikation in der Speditionslogistik die genannten Anforderungen (schnell, sicher, günstig, pünktlich) zuverlässig erfüllen können, gewinnen Vertrauen bei ihren Kunden und können sowohl die Kundenbindung erhöhen als auch ihren Marktanteil steigern. Folgende Probleme können bei der Kommunikation und Kooperation zwischen den verschiedenen Rollen innerhalb eines Speditionsunternehmens (Fahrer, Disponent, Kunde) auftreten und damit die Erfüllung der Anforderungen behindern [ErKo01]:

- Probleme des Disponenten:

- Diskontinuierlicher, mündlicher Informationsaustausch zwischen Disponent und Fahrer führt zu Zeitverzögerungen und Übertragungsfehlern.

- Der Fahrer ist aus Sicht des Unternehmens eine zentrale Informationsquelle - jedoch können die bei ihm auflaufenden Informationen bisher nicht ohne Medienbruch in die Speditionssoftware übernommen und damit nicht ohne weiteren manuellen Eingriff nutzbar gemacht werden (z.B. für einen Fuhrparkleitstand, für eine kunden-/prozessbezogene Statistik, für die Preiskalkulation).

- Aufgrund des fehlenden Kenntnisstandes der Disponenten über den Auftragsfortschritt der LKW-Fahrer ist eine kurzfristige Umdisposition nur begrenzt möglich.

- Termin- bzw. Soll-Abweichungen des LKW-Güterverkehrs, sofern diese bekannt sind, sind vom Disponenten nur beschränkt steuerbar bzw. zu korrigieren.

- Eine Kalkulation der Betriebs- bzw. Transportkosten ist nur mit zeitlicher Verzögerung zu erreichen.

- Probleme des Fahrers:

- Der Fahrer kann oft Störungen nur fernmündlich kommunizieren.

- Der Fahrer hat nur geringen Einfluss auf Tagestouren und Umdisposition.

- Der Disponent ist bei Rückfragen nicht ständig erreichbar.

- Probleme des Kunden:

- Der Auftragsfortschritt ist für den Kunden nicht transparent.

- Verspätungen sind nicht kalkulierbar.

Tabelle 1: Kommunikations- und Kooperationsprobleme

Durch den Wegfall einer standortgebundenen Erfassung und Übertragung von Informationen und Hinwendung zu einer mobilen Kommunikation, bei der die End- 
geräte nicht mehr ortsgebunden sind, sondern entweder selbst portabel sind oder durch eine Verbindung mit einem Fahrzeug eine Portabilität erlangen, können Informationen (Sensorikdaten, Telematikdaten, Dispositionsdaten) zeitnah übermittelt werden. Diese Informationen und deren Verarbeitung können zur Lösung der Probleme beitragen. Diese Lösungen betreffen dann nicht nur die dispositive Fahrzeugeinsatzsteuerung, sondern auch die strategische Fuhrparkplanung.

Softwaresysteme, welche die zeitnahe Erfassung und Übermittlung von Informationen unterstützen, werden als Kommunikationssysteme bezeichnet und immer zusammen mit Logistikanwendungen für Speditionsunternehmen von den verschiedensten Softwareherstellern angeboten. In Abhängigkeit verschiedener Faktoren, wie z.B. Art der transportierten Güter, bereits vorhandener mobiler Endgeräte oder dem Einsatz von Subunternehmern ist der Schwerpunkt bzgl. der Funktionalität bei diesen integrierten monolithischen Systemen unterschiedlich.

Diese Systeme nutzen zur Übertragung der Informationen die Technik GSM (Global System for Mobile Communication). „Die untersuchten Systeme realisieren die mobile Datenübertragung [...] ausschließlich über GSM. “ [SiEK03]. Durch die geringe Bandbreite dieser Technik (bis 9,6 kBit/s) können nur einfache Informationen (Texte) übertragen werden. Die Übertragung von audiovisuellen Informationen (Fotos, Videos) ist nicht - oder nur eingeschränkt - möglich. Dies führt dazu, dass auch die Anwendungen, die auf diesen integrierten monolithischen Systemen aufbauen, ebenfalls nur einfache Techniken nutzen (SMS, WAP). Die zukünftige Verbesserung der Bandbreite durch den Einsatz neuer Techniken (GPRS, $H S C S D, E D G E, U M T S$ ) führt daher nicht zwangsläufig zu einer Verbesserung der Anwendungen, da diese Anwendungen nicht auf die Nutzung der neuen Techniken ausgelegt wurden [Herz01].

Auf der Basis dieser neuen Kommunikationstechniken müssen daher sowohl neue Anwendungen entworfen und realisiert werden und durch Aufbrechen des monolithischen Ansatzes eine Trennung der Systeme erreicht werden. Bei dem erforderlichen softwaretechnischen Vorgehen können sowohl neue Programmiersprachen $(J A V A, C \#)$ verwendet werden als auch Plattformen (J2EE, .NET) für verteilte Client/Server-Architekturen genutzt werden.

\section{Das Verbundforschungsprojekt Mobile Spedition im Web-SpiW -}

Das vom BMBF geförderte Verbundprojekt Mobile Spedition im Web (SpiW) verfolgt daher drei wesentliche Ziele bei der Entwicklung eines Kommunikationssystems, welche durch das Projektkonsortium definiert wurden:

- Integration mit Speditionslogistikanwendungen, 
- Anpassung an unternehmensspezifische fachliche Geschäftsprozesse,

- Nutzung neuer Telematik- und Kommunikationstechniken.

Die Integration mit Speditionslogistikanwendungen erfolgt über generische Schnittstellen und bietet daher den Vorteil, dass Speditionslogistikanwendungen vieler Anbieter mit dem Kommunikationssystem integriert werden können. Es wird hierdurch eine Trennung zwischen kaufmännischen Dispositions- und Logistiksystemen und Kommunikationssystemen erreicht. Unternehmen sind dann nicht mehr gezwungen monolithische Softwaresysteme (z.B. Flottenmanagementsysteme [JuLa01]) mit einem umfangreichen Funktionsumfang zu kaufen und einzusetzen, nur um die Kommunikationsfunktionalität zu nutzen. Durch diese Trennung wird ebenfalls ein Investitionsschutz unterstützt, d.h. existierende Softwaresysteme können weiterhin genutzt und brauchen nicht ersetzt zu werden. Hierdurch soll die Möglichkeit - gerade für klein- und mittelständische Unternehmen - geschaffen werden, sich durch den Einsatz neuer Softwaresysteme am Markt zu behaupten.

Da die Arbeitsabläufe in jedem Speditionsunternehmen individuell sind, muss das Kommunikationssystem - gerade weil es als eigenständiges System zu einer existierenden Speditionslogistikanwendung eingesetzt werden soll - an diese Geschäftsprozesse angepasst werden. Um diese Anpassung ohne umfangreiche Programmiertätigkeiten durchführen zu können, wird bei der Entwicklung des Kommunikationssystems eine dynamische Geschäftprozesssteuerung realisiert (vgl. Kap. 6).

Die Übertragung von Informationen erfolgt durch neue Kommunikationstechniken (GPRS, EDGE, HSCSD, UMTS) mit Vorteilen wie z.B. eine dauerhafte Kommunikationsverbindung aufrechtzuerhalten oder komplexe Informationen (Bilder, Straßenkarten, Anfahrtsskizzen etc.) übertragen zu können. Zur Standortbestimmung und zur Verfolgung von Transporten können diese terrestrischen Techniken für ein „Tracking \& Tracing“ ebenso verwendet werden, wie satellitengestützte Techniken (z.B. GPS) [Mehl96]. Die terrestrischen Techniken werden durch die Mobilfunkunternehmen als sog. Location Based Services (LBS) [DaRW02] angeboten. Im Rahmen einer Kosten/Nutzen-Analyse muss der Einsatz der verschiedenen Dienste und Techniken gegeneinander abgewogen werden. Die Art und Weise der Nutzung dieser Dienste, die Integration eines Mauterfassungssystems, und der Zugriff auf die Sensorikdaten eines Fahrzeugs über den seriellen CAN-BUS findet sich in der Funktionalität eines Kommunikationssystems wieder [Kien94], [Lawr95].

Die Entwicklung dieses Kommunikationssystems erfolgt in diesem Verbundprojekt interdisziplinär, d.h. verschiedene akademische und industrielle Partner mit unterschiedlicher fachlicher Qualifikation arbeiten an speziellen Aspekten und an der Lösung von spezifischen Problemen. 
Es werden unter anderem die Arbeitsprozesse in Speditionsunternehmen analysiert und Verbesserungsvorschläge hierfür erarbeitet. Diese Analyse wird von Spezialisten aus dem Bereich der Arbeitsorganisationsforschung durchgeführt. Diese verbesserten Arbeitsprozesse fließen in die Entwicklung des Kommunikationssystems ein. Es findet aber auch eine Rückkopplung statt, d.h. es wird ebenfalls analysiert, welche Auswirkung der Einsatz eines Kommunikationssystems auf die Arbeitsprozesse hat. Durch die Kopplung von Arbeitsprozessen und Softwaresystemen ergeben sich sog. soziotechnische Systeme [HeKL02], die letztendlich in Unternehmen eingeführt werden.

Nicht nur die Arbeitsprozesse sondern auch die Logistikprozesse erfahren durch den Einsatz eines Kommunikationssystems eine Veränderung [ErKo01]. Durch eine verbesserte und zeitgenaue Kommunikation können zum Beispiel Güterumschläge oder Begegnungsverkehr vermieden oder reduziert werden. Die Analyse und Verbesserung der Logistikprozesse erfolgt durch Spezialisten aus diesem Bereich. Auch hier findet durch eine Analyse der Auswirkungen von verbesserten Logistikprozessen auf das Kommunikationssystem eine Rückkopplung statt.

Dieses Verbesserungs- /Rückkopplungsvorgehen erfordert eine inkrementelle und iterative Vorgehensweise bei der softwaretechnischen Entwicklung des Kommunikationssystems. Durch komponentenorientierte Paradigmen [GrTh00] wird diese Vorgehensweise unterstützt. Der Entwurf und die Realisierung des Systems erfolgt durch Spezialisten aus dem Bereich Angewandte Telematik.

Das Verbundprojekt hat eine Laufzeit von 30 Monaten und begann im November 2001. Beteiligt an diesem Verbundprojekt sind als akademische Partner die Universität Dortmund, die Universität Bremen und die Universität Leipzig und als industrielle Partner die Stute Verkehrs-GmbH in Bremen und die Dekra AG in Stuttgart.

\section{Anwendungsszenario}

Dem Projekt liegt folgendes Anwendungsszenario zugrunde. Im Rahmen eines Güterverkehrs wird ein Speditionsunternehmen beauftragt, bei einem Versender eine Anzahl von Ladungen abzuholen und auszuliefern. 


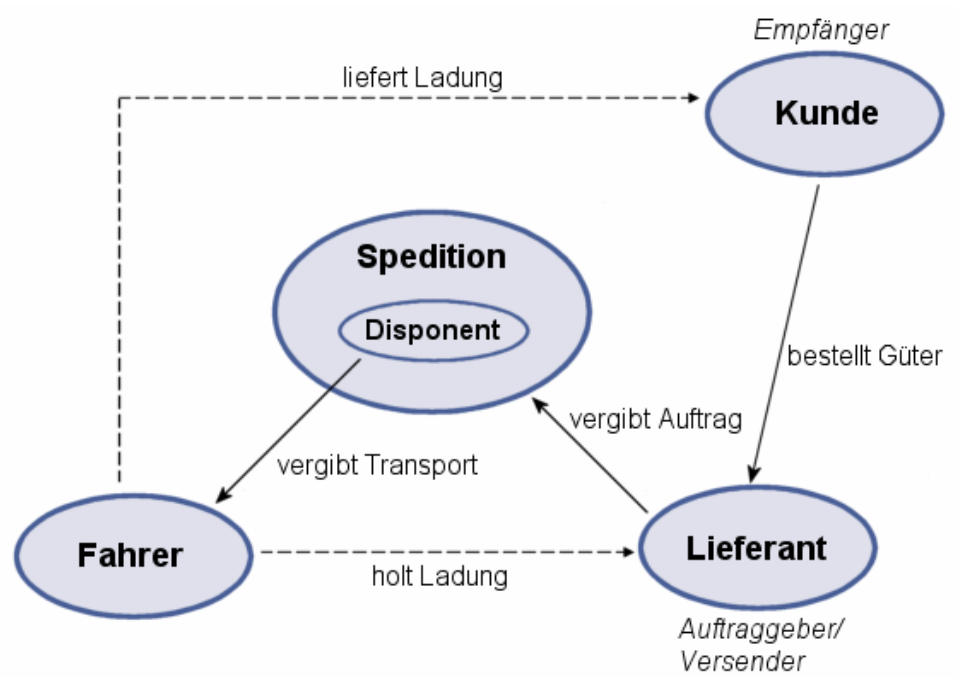

Abbildung 1: Rollen beim Güterverkehr

Das Speditionsunternehmen vergibt einen Transport an einen Fahrer - angestellt oder Subunternehmer -, der die Ladungen bei dem Versender abholt und nacheinander an die Empfänger abliefert (vgl. Abbildung 1).

Durch dieses Szenario wird ausschließlich der Ganzladungsverkehr und der Teilladungsverkehr (z.B. Transport von Stahl und Stahlerzeugnissen) beschrieben. Der Ganzladungs- und Teilladungsverkehr ist eine spezielle Variante des Güterverkehrs und unterscheidet sich im Vergleich zu den Sammelverkehren und KEPVerkehren (Kurier, Express und Paket-Verkehren) dadurch, dass kein Vor- oder Nachlauf stattfindet und dass die Kunden (Versender, Empfänger) keine Privatpersonen sondern Unternehmen sind.

Zur Unterstützung der Kommunikation und Kooperation zwischen den beteiligten Rollen in diesem Szenario werden technische Hilfsmittel und Softwaresysteme eingesetzt. Das Speditionsunternehmen setzt eine Speditionslogistikanwendung ein, um den Disponenten bei der Disposition von Aufträgen zu unterstützen. Die Kooperation und Kommunikation mit dem Fahrer erfolgt in der Regel mündlich per Telefon/Mobiltelefon oder schriftlich mittels Dokumenten (Tourenplänen, Lieferscheine, Ladelisten). Die Kommunikation zwischen dem Disponenten und den Kunden (Empfänger/Lieferanten) erfolgt telefonisch oder schriftlich per Fax oder mittels elektronischen Datenaustausches (EDI) über eine Schnittstelle der Speditionslogistikanwendung.

Bei der ausschließlichen Verwendung dieser technischen Hilfsmittel können die in Tabelle 1 genannten Probleme auftreten. Der Einsatz eines Kommunikationssys- 
tems als Ergänzung zu einer Speditionslogistikanwendung soll bei der Problemlösung helfen.

Die Benutzer eines Kommunikationssystems wären dann hauptsächlich die Disponenten und die Fahrer eines Speditionsunternehmens. Durch die Integration mit einer Speditionslogistikanwendung könnten Kunden einen Teil der Funktionalität des Kommunikationssystems nutzen (z.B. Tracking \& Tracing).

Es werden hier daher im Wesentlichen zwei Arten von Benutzern unterschieden, die auch verschiedene Geräte verwenden. Während der Fahrer eines Speditionsunternehmens ein mobiles Endgerät (PDA - Personal Digital Assistant) nutzt, verwendet der Disponent ein stationäres Endgerät ( $P C$-Personal Computer).

Durch einen Auftrag wird die Versendung von Gütern von ein oder mehreren Beladestellen zu ein oder mehreren Entladestellen definiert; bezogen auf einen Versender. Güter, die von den gleichen Be- und Entladestellen verladen werden sollen und aus rechtlichen, organisatorischen oder logistischen Gründen gemeinsam transportiert werden dürfen und können, werden zu einer Sendung zusammengefasst. Somit setzt sich ein Auftrag u.U. aus mehreren Sendungen zusammen. Eine Sendung bezeichnet aber keinen physischen Transport, sondern dient lediglich als logistisches Hilfsmittel während der Disposition. Wiederum abhängig von technischen oder rechtlichen Faktoren wird eine Sendung in einen oder mehrere Transporte unterteilt. Ein Transport ist schließlich die physische Realisierung einer Güterlieferung von einer Beladestelle zu einer Entladestelle durch ein Speditionsfahrzeug.

Ein Transport enthält eine Menge von Ladungspositionen. Eine Ladungsposition beschreibt ein Gut oder eine Menge von Gütern, die zu einer größeren Einheit (z.B. Palette) zusammengefasst sind.

Ein Speditionsfahrzeug kann mehrere Transporte gleichzeitig ausführen. Alle Transporte eines Speditionsfahrzeugs werden zu einer Tour bzw. Route zusammengefasst. Eine Tour ist eine geordnete Liste von durchzuführenden Transporten, die der Disponent einem Fahrer zuteilt. Die Reihenfolge dieser Transporte kann der Fahrer nach eigenem Ermessen ändern, wenn bestimmte Rahmenbedingungen dies erforderlich machen (z.B. Stau auf einer Autobahn, geringere Mengen von Gütern bei der Verladung). Die sich daraus ergebene Liste von Transporten wird dann als Route bezeichnet.

Da nur im Idealfall eine Route mit einer Tour identisch ist und sich die Tour dynamisch - durch die Beladung - ergibt und sich auch dynamisch - durch die Fahrt - ändern kann, ist eine zeitnahe Kommunikation und Kooperation zwischen dem Fahrer und dem Disponenten erforderlich, um eine Optimierung der Transporte zu erreichen.

Bei der Durchführung einer Route kann der Fahrer nach Erreichen einer Beladestelle feststellen, dass die Menge der durch den Lieferanten bereitgestellten Teilladungen nicht der Menge entspricht, die in dem Auftrag vermerkt sind. Der 
Abgleich der Informationen über die Teilladungen mit den Informationen im Auftrag kann entweder manuell oder über einen Barcodescanner erfolgen. Folgende Fälle können hier - exemplarisch - auftreten:

1. die Menge ist kleiner als vermerkt,

2. die Menge ist größer als vermerkt,

3. die Menge entspricht der vermerkten Menge, aber einzelne Teilladungen sind nicht korrekt.

$\mathrm{Zu}$ 1.)

Der Disponent wird über die Mindermenge elektronisch informiert ${ }^{2}$. Aufgrund der Information welche Art eines Speditionsfahrzeugs zurzeit eingesetzt wird, kann der Disponent - mit Hilfe einer Speditionslogistikanwendung - einen weiteren Transport bestimmen und dem Fahrer einen neuen Auftrag übermitteln.

$\mathrm{Zu}$ 2.)

Der Disponent wird über die Mehrmenge elektronisch informiert. Der Disponent entscheidet, ob der Fahrer die Kapazität seines Speditionsfahrzeugs ausnutzt und die möglichen Teilladungen lädt oder ob der Fahrer eine kurze Leerfahrt zu einer anderen Beladestelle durchführen soll. In letzteren Fall wird der Auftrag storniert und ein anderer Fahrer mit einem anderen größeren Speditionsfahrzeug erhält einen neuen aktualisierten Auftrag über die gesamte Menge. Im ersten Fall wird ein weiterer Auftrag erstellt und an einen anderen Fahrer übermittelt, der dann die noch ausstehenden Teilladungen abholt.

$\mathrm{Zu}$ 3.)

Beim Abgleich der Informationen über Teilladungen mit dem Auftrag wird durch den Fahrer festgestellt, dass einige Teilladungen falsch sind. Die Teilladungen sind entweder durch den Versender falsch zusammengestellt oder nach Rückfrage mit dem Empfänger neu zusammengestellt worden. Aufgrund der Informationen vor Ort

- identischer Empfänger,

- falscher Aufladepunkt in einem großen Werksgelände,

- nicht zustellbare Teilladung, da falsche Empfängerangaben

versucht der Fahrer zu entscheiden, ob diese nicht korrekten Teilladungen trotzdem geladen werden können oder eine Beladung ausgeschlossen werden muss. Wenn eine Entscheidung vor Ort durch den Fahrer nicht möglich ist, wird der

2 Anmerkung: Bei allen elektronisch übermittelten Informationen werden die Probleme bei der Datenübertragung oder fehlende oder ausstehende Empfangsbestätigung, etc. berücksichtigt. 
Disponent elektronisch informiert. Der Disponent entscheidet in ähnlicher Form wie bei 2).

Der Fahrer setzt seinen Transport fort (vgl. 1. oder 3.) und liefert anschließend die Teilladungen an die Entladestelle der einzelnen Empfänger oder liefert die Teilladungen sofort an einen Empfänger (vgl. 1.).

Der Empfang der Teilladungen wird durch den Empfänger elektronisch durch eine elektronische Unterschrift dokumentiert. Auch werden die Teilladungen überprüft, indem die Informationen des Auftrags mit den Informationen des Kunden verglichen werden. Dies erfolgt entweder manuell oder mittels Erfassung eines Barcodes. Bei Schäden an der Ladung - verursacht durch den Transport - wird bei Bedarf und vorhandenen technischen Gegebenheiten ein Foto oder ein Video elektronisch an den Disponenten übermittelt.

\section{Aspekte des Kommunikationssystems}

Aus der Verfolgung der genannten zwei wesentlichen Projektziele resultiert eine besondere Anforderung an das Kommunikationssystem: Das Kommunikationssystem muss in einem hohen Masse flexibel adaptierbar sein. Diese Flexibilität wird durch verschiedene Faktoren bestimmt, z.B.:

- Welche Art von Gütern werden durch ein Unternehmen transportiert?

Für Unternehmen, die auf den Transport von Flüssigkeiten spezialisiert sind, ist die Erfassung und Verarbeitung von Sensorikdaten (Temperatur, Druck) insbesondere wenn es sich um Gefahrgüter handelt - von besonderer Relevanz, während das Erfassen und das Verarbeiten von Barcodes bei der Auslieferung dieser Flüssigkeiten weniger wichtig ist.

- Welche Speditionslogistikanwendung wird durch ein Unternehmen eingesetzt? Von der Speditionslogistikanwendung hängt ab, welche Daten zwischen dem Kommunikationssystem und der Anwendung ausgetauscht werden können. Gerade bei kleineren Unternehmen (bis zu 5-6 Fahrzeugen) werden einfache Bürokommunikationsanwendungen - die keine explizite Disposition ermöglichen - eingesetzt. Hier ist die Art der Schnittstelle zwischen beiden Systemen von Bedeutung.

- Welche fachlichen Geschäftsprozesse sollen unterstützt werden?

Gerade bei größeren Unternehmen mit verschiedenen Niederlassungen im Bundesgebiet agieren diese Niederlassungen nicht alle gleich, sondern sind spezialisiert auf einen bestimmten Kundenkreis (z.B. Stahlerzeuger) oder eine bestimmte Güterklasse (z.B. Fahrzeugmotoren). Heraus ergeben sich unterschiedliche fachliche Geschäftsprozesse, die unterstützt werden sollen, z.B. 
kann eine elektronische Unterschrift zur Empfangsbestätigung möglich sein oder das Einlesen von Barcodes für Güter muss möglich sein.

Da während des Entwurfs und der Entwicklung des Kommunikationssystems nicht bekannt ist, welches Unternehmen das System später nutzen wird, muss bei der Modellierung der Geschäftsobjekte, bei der Erstellung der Systemarchitektur und der Festlegung der Schnittstellen sowie bei der Umsetzung von fachlichen Geschäftsprozessen das Kommunikationssystem konzeptionell für eine flexible Adaptierbarkeit vorgesehen werden (z.B. auch für eine Mehrsprachlichkeit).

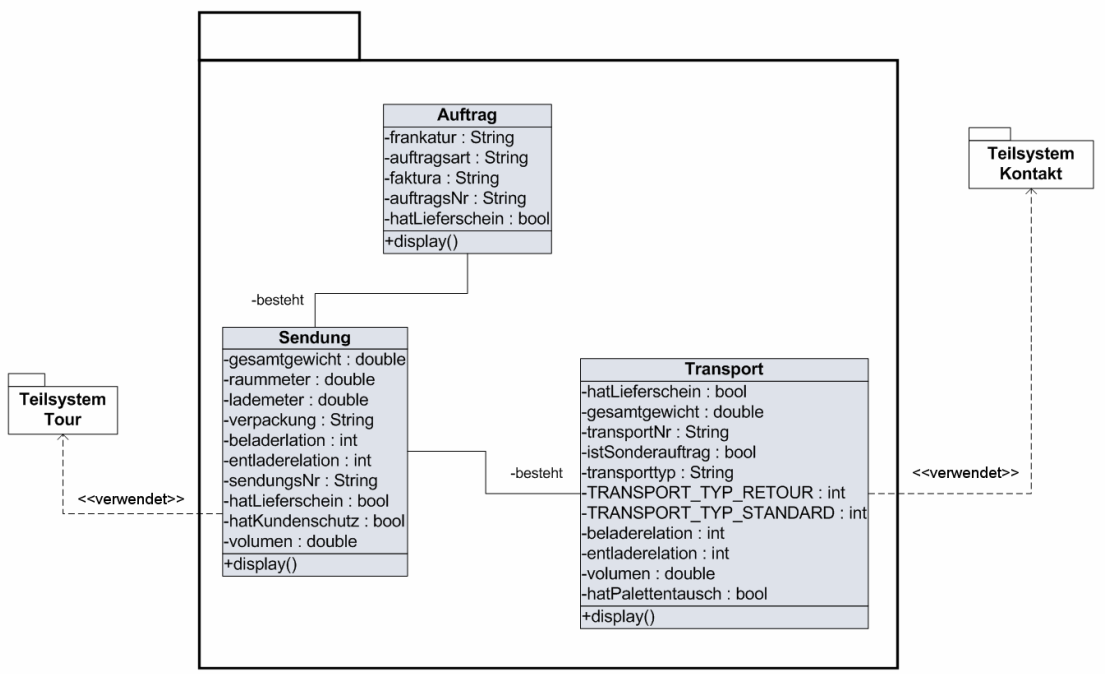

Abbildung 2: Teilsystem- und Klassenmodell

Bei der Modellierung werden alle fachlichen Geschäftsobjekte durch Verwendung der Unified Modeling Language (UML) als Klassen eines unternehmensweiten Klassenmodells modelliert. Durch diese fachlichen Geschäftsobjekte wird die fachliche Architektur eines Softwaresystems beschrieben [BaGe98]. Zur weiteren Strukturierung dieses unternehmensweiten Klassenmodells werden mehrere Klassen zu Teilsystemen (Packages) zusammengefasst. Einen Ausschnitt aus diesem Klassenmodell, das Teilsystem Auftrag mit den relevanten Geschäftsobjekten Auftrag, Sendung und Transport, zeigt die Abbildung 2. 


\section{Systemarchitektur des Kommunikationssystems}

Die Systemarchitektur des Kommunikationssystems besteht aus drei Komponenten: Mobilen Endgeräten, stationären Endgeräten und einem Anwendungsserver. Die mobilen Endgeräte nutzen ein leiterungebundenes Medium (GSM, EDGE, GPRS, HSCSD, UMTS) zur Kommunikation mit einem Anwendungsserver, während durch die stationären Endgeräte ein leitergebundenes Medium (FastEthernet, Ethernet) zur Kommunikation mit dem Anwendungsserver verwendet wird.

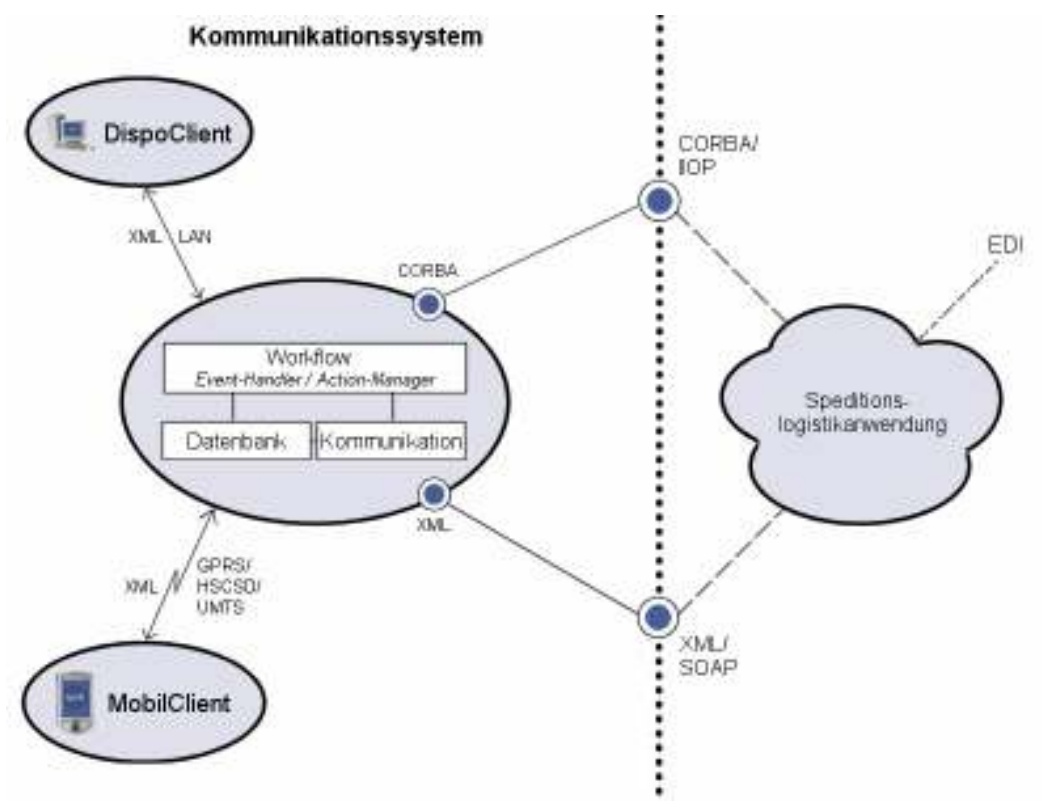

Abbildung 3: Architektur des Kommunikationssystems

Die Softwarearchitektur des Kommunikationssystems folgt dem Client/ServerKonzept [Lewa98], [OrHe96]. Durch einen Anwendungsserver wird die Geschäftslogik zur Bearbeitung von Geschäftsobjekten bereitgestellt. Zur Umsetzung, Unterstützung und Ausführung dieser fachlichen Geschäftslogik bedient sich der Anwendungsserver weiterer Serverkomponenten: Einem WorkflowServer, einem Kommunikations-Server und einem Datenbank-Server (vgl. Abbildung 3). Durch die Clients werden die vom Server entsprechend der fachlichen Geschäftslogik angebotenen Dienste genutzt, um Daten zielgerichtet einem Benutzer zur Bearbeitung zur Verfügung zu stellen.

Wenn die Geschäftslogik nicht nur durch einen Anwendungsserver bereitgestellt wird, sondern Teile der Geschäftslogik auf einen Client verlagert werden, werden Clients als Thick-Clients [Lewa98] bezeichnet. Dagegen verfügen Thin-Clients 
über keine eigene Geschäftslogik, sondern nutzen ausschließlich die von einem Anwendungsserver bereitgestellten Dienste.

Während Thin-Clients auf den stationären Endgeräten zur Anwendung kommen, kommen Thick-Clients auf den mobilen Endgeräten zur Anwendung. Dies liegt in der Tatsache begründet, dass nicht immer sichergestellt werden kann, dass die Kommunikationsverbindung über ein leiterungebundenes Medium zwischen mobilen Endgeräten und dem Anwendungsserver immer verfügbar ist. Die Wahrscheinlichkeit das eine Kommunikationsverbindung über ein leitergebundenes Medium in einem Local Area Network (LAN) über längere Zeit ausfällt ist gering, so dass bei stationären Endgeräten der Einsatz eines Thin-Client ausreichend ist. Um jedoch den Anforderungen, die aus den in der Einleitung genannten Problemen resultieren, gerecht zu werden, muss ein Teil der Geschäftslogik auch weiterhin ausgeführt werden können, wenn die Kommunikationsverbindung mal unterbrochen ist, was trotzdem den Einsatz eines Thick-Clients erforderlich macht. Nach Wiederherstellung der Kommunikationsverbindung muss eine Synchronisation stattfinden.

Die Art der Daten, die durch die verschiedenen Clients zur Bearbeitung zur Verfügung gestellt werden, ist unterschiedlich. Nicht jeder Benutzer benötigt aufgrund seiner Rolle die gleichen Daten. Während der Fahrer im Wesentlichen nur Transport- und Telematikdaten benötigt, ist der Disponent neben den Transportdaten auch an den Dispositionsdaten interessiert. Durch die verschiedenen Clients werden in Abhängigkeit von der Art des Endgeräts und dessen technischen Möglichkeiten (z.B. Größe des Displays), nicht nur die Daten unterschiedlich dargestellt, sondern auch die Menge der dargestellten Daten begrenzt. Ebenso ist die Art der Benutzungsführung unterschiedlich (z.B. durch vordefinierte Tasten beim $P D A$ oder Zeigersteuerung durch eine Maus beim $P C$ ).

Auf dem Markt für Speditionslogistikanwendungen hat sich zurzeit kein Anbieter oder Hersteller als markbestimmend herausgestellt ${ }^{3}$, wodurch die unterschiedlichsten Softwaresysteme bei Speditionsunternehmen eingesetzt werden. Durch diese Vielfalt der eingesetzten Speditionslogistikanwendungen ist es erforderlich, für das Kommunikationssystem einen Integrationsmechanismus $\mathrm{zu}$ entwickeln, der die folgenden Anforderungen erfüllt:

- Anpassbarkeit, d.h. unterschiedliche Speditionslogistikanwendungen können mit geringem Aufwand integriert werden,

- Flexibilität/Erweiterbarkeit, d.h. neue Anforderungen an das Kommunikationssystem müssen ohne Veränderung des Integrationsmechanismus umsetzbar sein.

3 Der Marktanteil des bekanntesten Anbieters von Softwaresystemen für Speditionsunternehmens liegt zurzeit bei ca. $8 \%$. 
Aufgrund dieser Anforderungen bietet es sich an, den Integrationsmechanismus entsprechend eines Event / Action-Models zu realisieren. In diesem Modell werden durch verschiedenartige Ereignisse (Events) eine oder mehrere Aktionen (Actions) initiiert. Durch eine Eingabe (z.B. Eingabe von Daten, Modifikation von angezeigten Daten, Erfassung von Unterschriften oder Barcodes, etc.) eines Benutzers auf einem mobilen Endgerät, einem stationären Endgerät oder durch die Speditionslogistikanwendung werden Ereignisse ausgelöst. Diese Ereignisse werden auf dem Anwendungsserver in einer Warteschlange (Event-Handler) aufgenommen. Die Steuerung der Ausführung von Aktionen wird jeweils von einem Action-Handler übernommen. Da die Ereignisse klassifiziert sind, können für jedes Ereignis oder für eine Klasse von Ereignissen bestimmte Aktionen definiert werden, die ausgeführt werden sollen (Versand einer Nachricht als SMS, E-Mail oder Fax, Anzeigen einer Statusmeldung durch die stationären / mobilen Clients oder der Speditionslogistikanwendung, Öffnen eines Informationsfensters durch die stationären / mobilen Clients oder der Speditionslogistikanwendung, Aufruf einer Funktion in einer Datenbank oder in der Speditionslogistikanwendung).

Dieser Integrationsmechanismus wird zweifach eingesetzt:

- Für den Austausch von Daten zwischen den verschiedenen Clients und dem Anwendungsserver

- Für den Austausch von Daten zwischen dem Anwendungsserver und der Speditionslogistikanwendung.

Im ersten Fall wird, um einen Austausch von Daten zwischen den Clients und dem Anwendungsserver zu gewährleisten, die Struktur jedes Geschäftsobjekts - unter Verwendung der Klassendiagramme - zunächst durch eine Document Type Definition (DTD) [BöU199] beschrieben. Der Austausch von den Geschäftsobjekten selbst erfolgt anschließend dann in Form von XML Daten [Tolk99]. Die Transformation von Objekten in XML Daten und umgekehrt erfolgt durch einen Parsing/Unparsing Vorgang auf der Seite der Clients als auch des Anwendungsservers. Dieser Parsing/Unparsing Vorgang ist erforderlich, da die Realisierung des Anwendungsservers und der Clients entsprechend eines Komponentenmodells zusammen mit einer objektorientierten Programmiersprache erfolgt. In den Implementierungen der Clients ist jeweils ein Event-Handler und ein Action-Manager realisiert.

Im zweiten Fall wird dieser Mechanismus technisch durch die Verwendung einer CORBA Implementierung (Common Object Request Broker Architecture) [Linn98] realisiert. Hier wird im einfachsten Fall sowohl der Event-Handler als auch der Action-Manager als jeweils ein Dienst realisiert. Je nach Komplexität kann der Mechanismus derart erweitert werden, das z.B. der Action-Manager als eine Menge von einzelnen Diensten realisiert wird, wobei jeder Dienst auf ein Ereignis oder eine Klasse von Ereignissen reagiert. Ebenso ist es möglich, den $E$ vent-Handler als eine Menge von Diensten zu realisieren, wobei jeder Dienst eine Warteschlange für ein Ereignis oder eine Klasse von Ereignissen repräsentiert. Bei 
einer Verwendung von verschiedenen CORBA Implementierungen, für den EventHandler und für den Action-Manager, können diese Dienste über das Internet Inter-ORB Protocol (IIOP) [Linn98] miteinander kommunizieren. So kann z.B. für das Kommunikationssystem die CORBA Implementierung JBuilder 7.0 der Firma Borland eingesetzt werden, während für die Speditionslogistikanwendung der Domino Server der Firma Lotus eingesetzt wird. Darüber hinaus können auch Implementierungen der Enterprise Java Beans Spezifikation der Firma Sun als Application Server eingesetzt werden, denn auch diese Server unterstützen das Internet Inter-ORB Protocol. Der Einsatz von Enterprise Java Beans Server ist insbesondere für die Unterstützung des Enterprise JavaBeans Component Models [GrSc02] interessant.

Eine weitere Möglichkeit zur Realisierung des Integrationsmechanismus für den Austausch von Daten zwischen dem Anwendungsserver und der Speditionslogistikanwendung kann durch Verwendung des Simple Object Access Protocol (SOAP) [Ches01], [Jeps01] erfolgen.

\section{Entwicklung des Kommunikationssystems}

Die softwaretechnische Architektur des Kommunikationssystems muss ein Höchstmaß an Flexibilität zur Gestaltung von fachlichen Geschäftsprozessen bieten. Fachliche Geschäftsprozesse müssen für spezielle und individuelle Profile (Kundenprofile, Standortprofile, etc.) dynamisch beschrieben und ebenso an neue fachliche Änderungen dynamisch angepasst werden können. Diese Flexibilität wird durch die komponentenbasierte Vorgehensweise bei der Entwicklung des Kommunikationssystems erreicht.

Die fachlichen Geschäftsprozesse, die durch das Kommunikationssystem unterstützt werden sollen, werden durch sog. Workflows beschrieben (vgl. Abbildung 4). Durch jeden Workflow wird genau ein fachlicher Geschäftsprozess (z.B. Verweigerung einer Auftragsnahme) mit seinen Bedingungen und Entscheidungen beschrieben. Fachliche Geschäftsprozesse können zusammenhängen, d.h. sie können sich initieren oder bedingen (z.B. die Erfassung einer Annahmeverweigerung durch einen Fahrer bei einem Kunden löst durch eine Änderung des Auftragsstatus eine Benachrichtigung an den Disponenten aus und damit kann durch ihn die Erstellung eines Retourenauftrags durchgeführt werden.) 


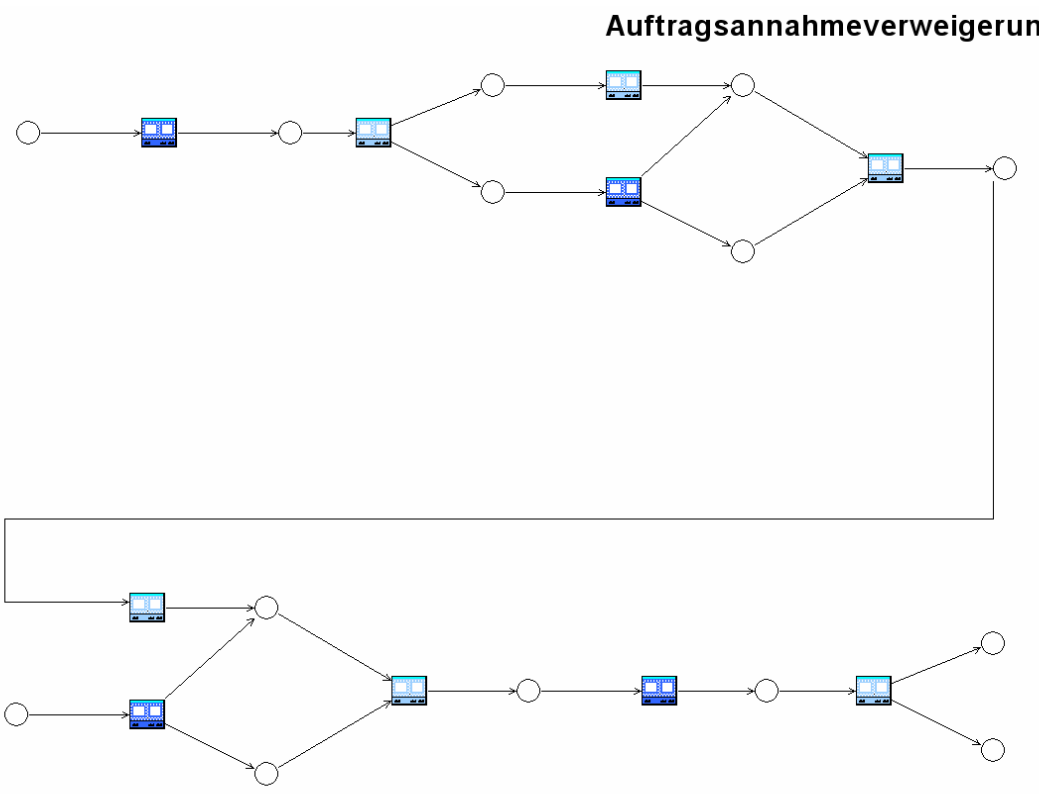

Statusänderung mit Benachrichtigung

Abbildung 4: Fachliche Geschäftsprozesse

Für jedes Teilsystem der fachlichen Architektur (vgl. Abbildung 5) werden ein oder mehrere Workflows beschrieben, durch den die fachlichen Daten erzeugt, bearbeitet und angezeigt werden können, z.B. die in der Abbildung 4 dargestellten Workflows sind dem Teilsystem Auftrag zugeordnet.

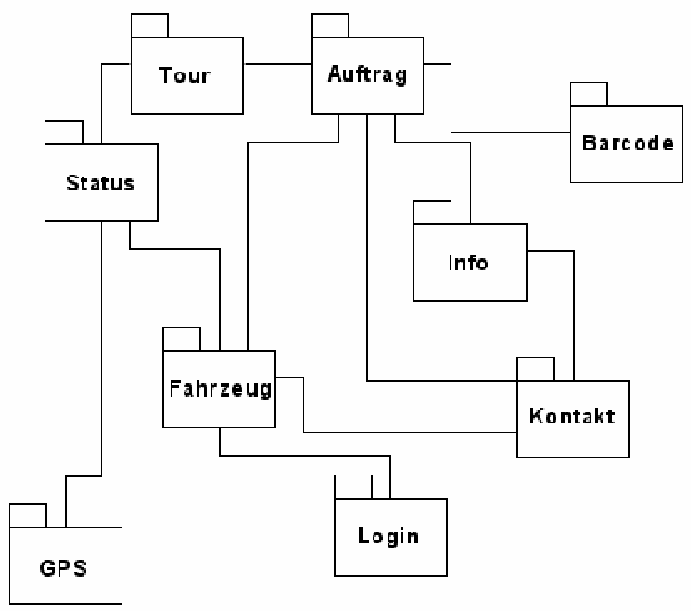

Abbildung 5: Teilsysteme des Kommunikationssystems 
Entsprechend der Abhängigkeiten zwischen Teilsystemen können verschiedene fachliche Workflows unterschiedlicher Teilsysteme ebenfalls voneinander abhängig sein. Diese Abhängigkeiten sind nicht immer fest, sondern können sich erst nach der Modifikation oder Erstellung von Daten ergeben (Entscheidung), u.U. auch erst nach dem Lesen von Daten.

Jede Klasse eines Teilsystems enthält eine Display-Methode (vgl. Abbildung 2). Mit dieser Methode werden die Daten einer Klasse - unter Berücksichtigung eines Style-Guides und entsprechender Benutzungsführung - dargestellt. Hierdurch wird sichergestellt werden, dass die Darstellung von Daten sowohl auf den mobilen Endgeräten als auch auf einem stationären Endgerät immer in der gleichen Art und Weise erfolgt und dass die einheitliche Benutzerführung zur Bearbeitung der Daten realisiert wird.

In Ergänzung zu den globalen Workflows, durch den fachliche Geschäftsprozesse beschrieben werden, beschreibt ein lokaler Workflow, den Ablauf von Methoden einer Klasse. Dieser lokale Workflow wird durch Realisierung der DisplayMethode einer Klasse bestimmt.

Jede Klasse eines Teilsystems wird als Komponente realisiert und implementiert. Bei dieser Realisierung werden die Schnittstellen für die Komponente festgelegt. Durch die Komponente werden die Daten gekapselt und durch Verwendung einer Datenhaltung dauerhaft gespeichert. Diese Realisierung der Komponenten erfolgt unter Verwendung einer objektorientierten Programmiersprache (JAVA, C\#).

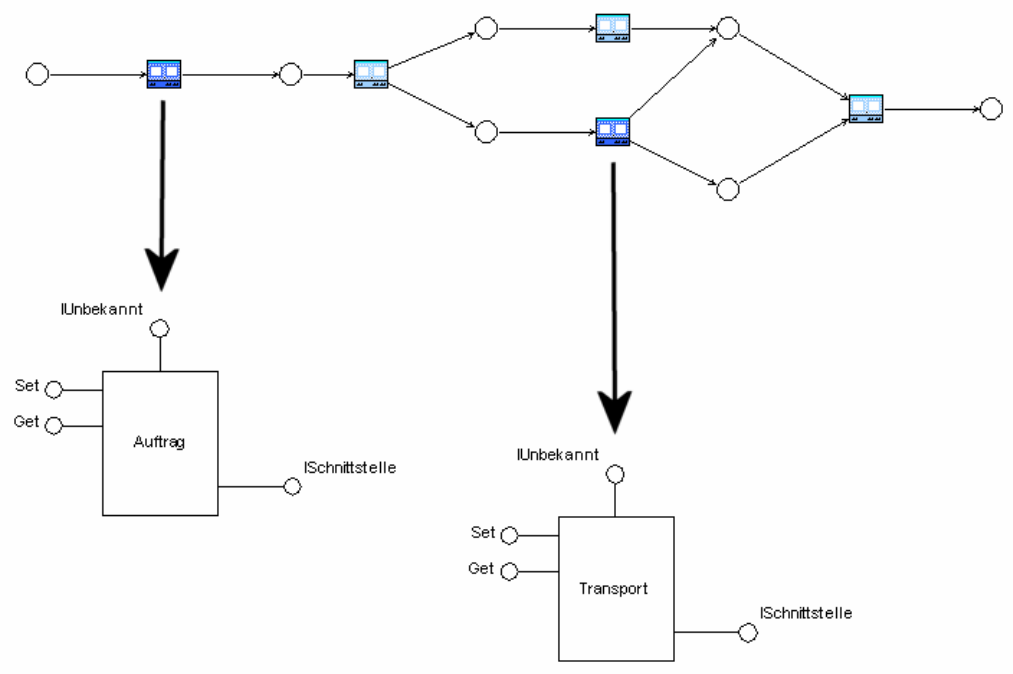

Abbildung 6: Realisierte und zugeordnete Komponenten 
Den Komponenten werden Tätigkeiten eines Workflows zugeordnet (vgl. Abbildung 6). In der Regel sollten Tätigkeiten jeweils nur einer Komponente zugeordnet werden und nur in Ausnahmefällen kann aber eine Zuordnung von mehreren Komponenten erfolgen. Eine derartige komplexe Zuordnung soll aber nur dann stattfinden, wenn eine fachliche Restrukturierung des Workflows nicht möglich ist. Der Workflow zwischen den zugeordneten Komponenten muss dann explizit - zusätzlich zu den globalen Workflows und den einzelnen lokalen Workflows - beschrieben werden.

Wenn bei der Durchführung einer Tätigkeit eine Entscheidung - auf der Basis von Daten - getroffen werden muss, die den weiteren Ablauf eines Workflows bestimmt, muss für die entsprechende Tätigkeit eine Entscheidungstabelle angegeben werden (vgl. Abbildung 7).

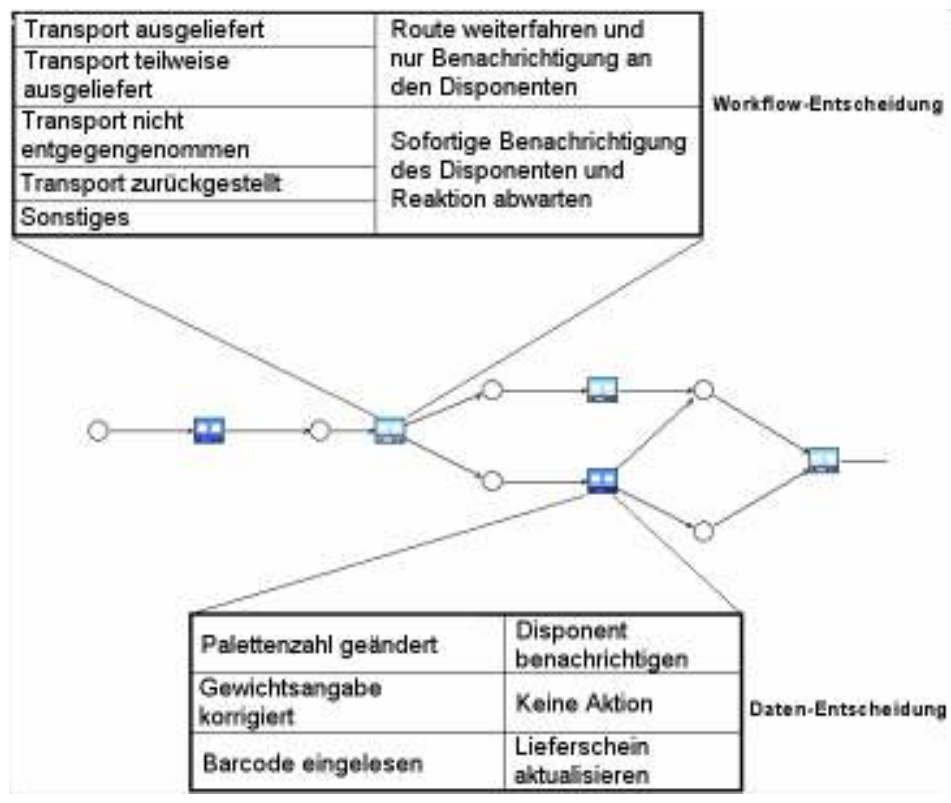

Abbildung 7: Entscheidungstabellen für Tätigkeiten

Die Steuerung der Workflows erfolgt durch einen Workflow-Server, der Teil des Anwendungsservers ist. Da Bestandteile eines fachlichen Geschäftsprozesses verteilt - sowohl auf den Clients als auch auf dem Anwendungsserver - ausgeführt werden, muss der Workflow-Server auch die verteilte Ausführung von Komponenten unterstützen und die Konsistenz und Integrität sicherstellen. Diese verteilte Ausführung kann zentral oder dezentral gelöst werden [GrGr95]. Unter Berücksichtigung der leiterungebundenen Kommunikation zwischen den mobilen Clients und dem Anwendungsserver - und den daraus resultierenden Problemen (Verbindungsabbruch, Verbindungsverfügbarkeit, etc.) -, bietet sich eine dezentrale Lö- 
sung an, bei der sowohl auf den mobilen Clients als auch auf dem Anwendungsserver jeweils ein Workflow-Server eingesetzt wird, die jedoch in der Mächtigkeit ihrer Funktionalität verschiedenen sind (vgl. Thin- / Thick-Clients). Die Frage, ob Komponenten zur Ausführung auf den mobilen Clients dynamisch nachgeladen werden können / müssen oder ob die Komponenten auf den Clients dauerhaft verfügbar sein sollten, wird durch eingesetzte Kommunikationstechnik (HSCSD, GPRS, EDGE, UMTS) - die das einsetzende Unternehmen vorgibt - bestimmt.

\section{Prototypische Anwendung}

Eine prototypische Anwendung kann dazu dienen den Nachweis der Praktikabilität von Ideen (Architektur und Vorgehen) zu liefern sowie eine Hard- und Softwareinfrastruktur auf ihre mögliche Verwendbarkeit in einem Softwareentwicklungsprojekt zu ermitteln. Diese Ermittlung kann von einer reinen Kosten/Nutzenbetrachtung über die Gestaltung einer Benutzungsoberfläche incl. Benutzerführung bis zu einer ausschließlichen technischen Machbarkeit reichen.

Die Grundlage dieses Prototyps ist das eingangs beschriebene Anwendungsszenario (vgl. Kapitel 2). Diese prototypische Anwendung wurde in Workshops verschiedenen Personen, die unterschiedliche Rollen (Fahrer, Disponent) innerhalb eines Speditionsunternehmens einnehmen, vorgestellt. Eine Auswertung der Ergebnisse hinsichtlich der Benutzungsoberfläche und Benutzerführung ist abgeschlossen [HaRi02]; eine Auswertung der Ergebnisse bzgl. des verwendetes Endgeräts und der genutzten Kommunikationstechnik steht noch aus.

Dieser Prototyp unterstützt zurzeit folgende Funktionalität: Der Fahrer führt eine Anmeldung auf dem mobilen Endgerät aus. Die personenspezifische Benutzeridentifikation wird an den Anwendungsserver übertragen und auf Plausibilität überprüft. Der Fahrer erhält eine positive oder negative Rückmeldung bei erfolgter Anmeldung. Nach korrekter Rückmeldung kann der Fahrer offene Aufträge einsehen und aus der Liste der offenen Aufträge ein oder mehrere Aufträge auswählen und so die Bearbeitung eines Auftrags übernehmen. Der Disponent erhält eine Bestätigung dieser Annahme (siehe Tabelle 2: Prototypische Anwendung). 

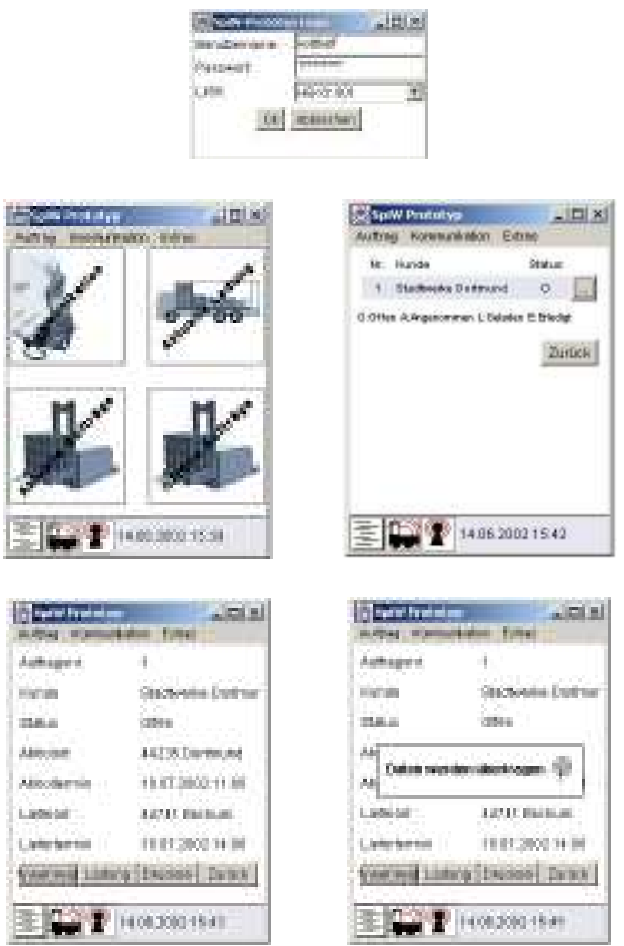

Tabelle 2: Prototypische Anwendung

Der Disponent kann jederzeit weitere Aufträge an einen Fahrer übermitteln, die auf dem mobilen Endgerät bis zur Annahme als offene Aufträge gekennzeichnet sind. Bei der Übernahme der Ladung an einer Beladestelle werden die Barcodes, die in einem angenommenen Auftrag ausgewiesen sind, mit den Barcodes der aufzunehmenden Ladung - entweder durch automatische Erfassung oder manuell verglichen. Der Status dieser Aufträge wird nach erfolgreicher Beladung von „offen“ auf ,,aktuell geladen“ gesetzt. Diese Statusänderung wird dem Disponenten elektronisch mitgeteilt. Nach erfolgter Lieferung an den Empfänger bestätigt dieser den Empfang der Lieferung durch eine Unterschrift auf dem mobilen Endgerät. Die ordnungsgemäße Abwicklung des Auftrags wird dem Disponenten elektronisch mitgeteilt. Bei Problemen, die bei der Entladung auftreten (Mindermenge, Annahmeverweigerung, Teilabnahme, etc.) wird der Disponent elektronisch informiert. Er kann weitere Maßnahmen ergreifen, z.B. Telefonate mit dem Versender oder Empfänger führen und dem Fahrer weitere Handlungsanweisungen geben. 
Der Disponent verfolgt auf der Basis der kontinuierlich übermittelten Informationen den Stand des Transports und kann diese bei weiteren Dispositionen berücksichtigten sowie Kunden und Lieferanten zeitnah Informationen über den aktuellen Auftragsfortschritt ihres Transports geben.

\section{Zusammenfassung und Ausblick}

Eine elektronische Unterstützung der Transportdurchführung ist aus informationstechnischer Sicht bisher nur schwach mit Speditionslogistikanwendungen integriert. Eine Kommunikation während der Transportdurchführung bzw. bei seiner Nachbereitung findet per Papier mittels Formularen oder per Telefon statt, was wiederum eine nachträgliche elektronische Erfassung von Informationen bedingt. Diese nachträgliche Erfassung ist fehlerbehaftet. Der Disponent ist während der Transportdurchführung nicht ausreichend über den Fortschritt informiert und muss sich bei Bedarf erforderliche Informationen aktiv beschaffen.

Es besteht daher einerseits der Bedarf an einer bidirektionalen Kommunikation zum zeitnahen Austausch von Informationen für die Transportdurchführung und Transportplanung zwischen den Fahrern und dem Disponenten und andererseits der Bedarf an einer unidirektionalen Kommunikation zwischen den mobilen Endgeräten und anderen nachgelagerten Softwaresystemen (Fibu, Fuhrparkplanung, etc.) zum Austausch von Informationen (Rechnungsdaten, Sensorikdaten, etc.) über ein stabiles leiterungebundenes Medium.

An die Hardware von mobilen Endgeräten in der Speditionslogistik werden besondere Anforderungen gestellt. Diese Anforderungen sind nicht identisch mit den Anforderungen an PDA's wie sie in der Bürokommunikation eingesetzt werden. Diese Geräte müssen robuster und fehlertoleranter hinsichtlich mechanischer Einflüsse sein (vgl. IP-Spezifikation). Des weiteren müssen sie durch zusätzliche Einund Ausgabegeräte erweiterbar sein (z.B. Barcodescanner, Kamera) und über eine Schnittstelle zum CAN-Bus eines Fahrzeugs verfügen.

Eine weitere aktuelle Frage, die zur Zeit noch nicht beantwortet werden kann und während der Projektlaufzeit beantwortet werden soll, ist die Art der Integration von mobilen Endgeräten mit den neuen Mauterfassungsgeräten für Lastkraftwagen (Hardware- oder Softwareintegration).

Trotz des zu erwartenden Nutzens eines integrierten Kommunikationssystems für die Speditionslogistik spielen die Kosten für die Anschaffung und - wichtiger noch - für den Betrieb eine zentrale Rolle bei der Konzeption und Realisierung.

Durch die industriellen Partner in dem Verbundprojekt soll sichergestellt werden, dass einerseits diese Aspekte bei der Konzeption und Entwicklung des Kommunikationssystems auch kontinuierlich berücksichtigt werden und andererseits eine 
weitere Nutzung und Vermarktung des Systems über das Ende des Projekts hinaus bei Bedarf gewährleisten werden kann.

\section{Literatur}

[BaGe98] Baker, Sean; Geraghty, Ronan: Java For Business Objects. In: Carmichel, Andy (ed.): Developing Business Objects, SIGS, Cambridge University Press, 1998, pp. 225237

[BöU199] Böhnlein, Ulrich; Ulbrich vom Ende, Achim: XML - Extensible Markup Language. In: Wirtschaftsinformatik, Band 41, Heft 3, Vieweg Verlag Wiesbaden, 1999, pp. 275-277

[Ches01] Chester, Timothy: Cross-Platform Integration with XML and SOAP. In: IT Professional, Vol. 3, Issue 5, IEEE Computer Society Press, 2001, pp.26-34

[DaRW02] Dao, Diep; Rizos, Chris; Wang, Jinling: Location Based Services: Technical and Business Issues. In: GPS Solutions, Vol. 6, No. 3, Springer Verlag, 2002, pp. 169178

[ErKo01] Erkens, Elmar; Kopfer, Herbert: WAP-LOG: Ein System zur mobilen Fahrzeugeinsatzsteuerung und Auftragsfortschrittkontrolle. In: Grünert, Sebastian: Logistik Management - Supply Chain Management und e-Business, Teubner Verlag, Stuttgart, 2001, pp. 293-303

[ErWa97] Ernst, Matthias; Walpukis, Dirk: Telekommunikation und Verkehr, Verlag Franz Vahlen, München, 1997

[GrGr95] Gruhn, Volker; Graw, Günther: Support of Cooperating and Distributed Business Process. In: Schäfer, W.; Botella, P. (eds.): Software Engineering - ESEC'95, Springer Verlag, 1995, pp. 8-27

[GrTh00] Gruhn, Volker; Thiel, Andreas: Komponentenmodelle, Addison Wesley, München, 2000

[GrSc02] Gruhn, Volker; Schneider, Andreas: EJB 2.0 Anwendungen, Addison Wesley, München, 2002

[HaRi02] Haltermann, Tobias; Ridder, Sebastian: Usability-Studie des Prototyps. Internes Arbeitspaket AP I.3/1 des Projekt SpiW, 2002

[Herz01] Herzig, Markus: Basistechnologien und Standards des Mobile Business. In: Wirtschaftsinformatik, Band 43, Heft 4, Vieweg Verlag, 2001, pp. 397-404

[HeKL02] Hermann, Thomas; Kunau, Gabriele, Loser, Kai-Uwe: Sociotechnical Walkthrough - ein methodischer Beitrag zur Gestaltung soziotechnischer Systeme. In: Herczeg, W.; Prinz, H. Oberquelle (Hrsg.): Mensch \& Computer, 2002: Vom interaktiven Werkzeug zu kooperativen Arbeits- und Lebenswelten, Stuttgart: Teubner Verlag, 2002, pp. 323-332 
[Jeps01] Jepsen, Tom: SOAP Cleans up Interoperability Problems on the Web. In: IT Professional, Vol. 3, Issue 1, IEEE Computer Society Press, 2001, pp.52-55

[JuLa01] Jung, Jürgen; Laak, Bodo van: Flottenmanagementsysteme - Grundlegende Technologien, Funktionen und Marktüberblick. Arbeitsbericht Nr. 28 des Instituts für Wirtschaftsinformatik der Universität Koblenz-Landau, 2001

[Kien94] Kiencke, Udo: Controller Area Network - From Concept to Reality. In: Proceedings of $1^{\text {st }}$ International CAN Conference, CAN in Automation (CIA), Erlangen, 1994, pp. 2-16

[Lawr95] Lawrenz, Wolfhard: Woldwide Status of CAN - Present and Future. In: Proceedings of $2^{\text {nd }}$ International CAN Conference, CAN in Automation (CIA), Erlangen, 1995, pp. 2-16

[Lewa98] Lewandowski, Scott: Frameworks for Computer-Based Client/Server Computing. In: ACM Computing Surveys, Vol. 30, No. 1, ACM Press, 1998, pp. 3-27

[Linn98] Linnhof-Popien, Claudia: Corba Kommunikation und Management, Springer Verlag, Heidelberg, 1998

[Meh196] Mehl, Horst: Global Positioning System. In: Informatik Spektrum, Band 19, Heft 1, Springer Verlag Heidelberg, 1996, pp.33-34

[OrHe96] Orfali, Robert; Harkey, Dan; Edwards, Jeri: The Essential Client/Server Survival Guide. Wiley Publ., 1996

[PoVe01] Posegga; Jocahim, Vetter, Simon: Wireless Internet Security. In: Informatik Spektrum, Band 24, Heft 6, Springer Verlag, 2001, pp. 383-386

[SiEK03] Siek, Katja; Erkens, Elmar; Kopfer, Herbert: Marktübersicht über Systeme zur Fahrzeugkommunikation im Straßengüterverkehr. Submitted to: Logistik Management, 5. Jahrgang, Ausgabe 2, Germa Press Verlag GmbH, Hamburg, 2003

[Tolk99] Tolksdorf, Robert: XML und darauf basierende Standards. In Informatik Spektrum, Band 22, Heft 6, Springer Verlag, Heidelberg, 1999, pp. 407-421 